Background The risk of Pelvic Inflammatory Disease (PID) and Ectopic Pregnancy (EP) from Chlamydia are crucial in estimating the cost-effectiveness of screening, but they remain poorly understood. Methods We use evidence from RCTs of screening and controlled observational studies to estimate the risk of PID following Chlamydia and the probability PID would be prevented by annual testing. The studies are synthesised using a model that allows for the possibility that the rate of developing PID is higher in the period soon after infection. We examine the role of Chlamydia and PID in EP using prospective evidence from the Lund study, evidence on the incidence and cumulative incidence of PID and EP in England, and retrospective evidence from case control studies. We assess the consistency of the data under different sets of assumptions about the severity of undiagnosed and non-hospital referred PID.

Results If the risk of PID due to Chlamydia is constant over time then the probability that an untreated Chlamydia episode causes clinical PID is estimated to be about $15 \%$, and there is approximately a $60 \%$ chance that annual testing would prevent an associated PID in a woman who becomes infected. If the PID rate is assumed to be higher for 1-3 months the respective figures are $16 \%$ and $50 \%$. We estimate that between a third and a half of EPs are caused by PID. Of these, around a third are due to Chlamydia though estimates are highly uncertain. Our comparison of different data sources suggests that undiagnosed PID carries some risk of EP. Conclusions Our findings support a public health strategy that (a) identifies women with Chlamydia as soon after infection as possible i.e. to get tested on change of sexual partner; (b) has a low threshold for diagnosing and treating women with pelvic pain or suspected PID.

\section{P3.014 THE IMPORTANCE OF AGE IN THE ROLE OF CHLAMYDIA IN THE ETIOLOGY OF PELVIC INFLAMMATORY DISEASE}

doi:10.1136/sextrans-2013-051184.0474

${ }^{1}$ M Price, ${ }^{2} \mathrm{~A}$ Ades, ${ }^{2} \mathrm{~N}$ Welton, ${ }^{3 \mathrm{~K}}$ Soldan, ${ }^{2 \mathrm{P}}$ Horner. 'University of Birmingham, Birmingham, UK; 2University of Bristol, Bristol, UK; ${ }^{3}$ Health Protection Agency, London, UK

Background Although the importance of age in the prevalence of Chlamydia is well recognised, its importance in the relationship between Chlamydia and pelvic inflammatory disease (PID) has received little attention in epidemiology.

Methods We generate and compare several sets of estimates of the population attributable fraction (PAF) of PID due to chlamydia by age-group using a number of data sources. Estimates are obtained using data from case-control studies and Chlamydia population prevalence in England. A second set of estimates is obtained from data on the incidence of PID, the incidence of Chlamydia, and the risk that a Chlamydia infection causes PID. We estimate the incidence of all-cause PID by age in England from routine data sources, and evidence on the proportion of PID episodes that are diagnosed. We synthesise these data with data from the control arm of the POPI trial. We estimate Chlamydia incidence by age in a multiparameter evidence synthesis of studies of Chlamydia incidence, prevalence, and duration of infection. Finally we estimate the risk of PID following Chlamydia from a statistical synthesis of randomised controlled trials using a multistate model. A third estimate of the PAF is obtained for the POPI trial population.

Results Prospective estimates of the PAF fall from 50\% (15\%, $100 \%)$ in women aged $16-19$ to $20 \%(6 \%, 49 \%)$ in women aged $35-44$, and estimates from retrospective data drop from $34 \%(17 \%, 55 \%)$ to $6 \%(2 \%, 14 \%)$. Changes with age groups are most likely due to changes in the aetiology of PID, but part of the effect would be explained if the risk of PID due to CT, or proportion of PIDs that are diagnosed, increases with age.

Conclusions The PAF of PID due to Chlamydia reduces dramatically with age. More attention needs to be given to age when designing and reporting results from epidemiological studies.

\section{P3.015 ESTIMATING THE POPULATION PREVALENCE OF CHLAMYDIA IN EUROPE: SYSTEMATIC REVIEW AND META-ANALYSIS}

doi:10.1136/sextrans-2013-051184.0475

${ }^{1} \mathrm{~S}$ Redmond, ${ }^{2} \mathbf{S}$ Woodhall, ${ }^{3,4} \mathrm{~J}$ van Bergen, ${ }^{5} \mathrm{H}$ Ward, ${ }^{6} \mathrm{~A}$ Uusküla, ${ }^{7} \mathrm{~B}$ Herrmann, ${ }^{8} \mathrm{~B}$ Andersen, ${ }^{9} \mathrm{H}$ Götz, ${ }^{10} \mathrm{O}$ Sfetcu, ${ }^{1} \mathrm{~N}$ Low. ${ }^{1} / n s t i t u t e$ of Social and Preventive Medicine, University of Bern, Bern, Switzerland; ${ }^{2}$ Health Protection Agency, London, UK; ${ }^{3}$ National Institute of Public Health (RIVM), Amsterdam, The Netherlands; ${ }^{4}$ STI/AIDS Foundation and University of Amsterdam, Amsterdam, The Netherlands; ${ }^{5} / m p e r i a l$ College London, London, UK; ${ }^{*}$ University of Tartu, Tartu, Estonia; ${ }^{7}$ University of Uppsala, Uppsala, Sweden ${ }^{8}$ Randers Regional Hospital, Randers, Denmark; ${ }^{9}$ Municipal Health Service, Rotterdam, The Netherlands; ${ }^{10}$ European Centre for Disease Control and Prevention, Stockholm, Sweden

Background Accurate information about the prevalence of Chlamydia trachomatis infections is needed to assess prevention and control measures at national and regional level.

Methods Systematic review up to August 2012 of populationbased cross-sectional studies that estimated chlamydia prevalence in high income countries, focussing on EU/EEA Member States. Data were extracted about study findings; the risk of bias was assessed and meta-analysis was performed where appropriate. Meta-regression was used to examine the relationship between chlamydia prevalence estimates and study response rates.

Results 25 population-based studies were included from $10 \mathrm{EU} /$ EEA countries and 14 studies from 6 other high income countries. Four EU/EEA Member States reported on nationally representative surveys of sexually experienced adults $\leq 26$ years, with response rates from $52-71 \%$. Chlamydia point prevalence estimates in 18-24 year olds (3 studies) ranged from $3.0-4.7 \%$ in women and from $2.4-4.7 \%$ in men. Chlamydia prevalence estimates in EU/EEA countries and other high income countries were statistically consistent. The combined estimate from 5 studies in 18-26 year olds in Europe and the USA was 4.3\% (95\% CI 3.7, $5.0 \%)$ in women and $3.6 \%(95 \%$ CI $2.9,4.3 \%)$ in men. In most studies there was either a high risk of selection bias in the methods used or insufficient information to judge. Estimates of chlamydia prevalence were inversely associated with response rate $(p=0.005$ in women, 0.011 in men).

Conclusions Selection bias in chlamydia prevalence surveys is likely, with over-estimation of prevalence being more likely than under-estimation. Cross-sectional surveys with lower response rates are associated with higher estimates of chlamydia prevalence. In studies with low response rates the percentage of chlamydia positive tests should not be interpreted as an estimate of population prevalence. Applying standards for the reporting of prevalence surveys might help to improve consistency in future.

\section{P3.016 PREVALENCE AND CORRELATES OF RECTAL-ONLY CHLAMYDIA INFECTION AT TWO CANADIAN STI CLINICS}

doi:10.1136/sextrans-2013-051184.0476

1J Gratrix, ${ }^{2} \mathrm{~J}$ Bergman, ${ }^{3} \mathrm{C}$ Egan, ${ }^{2} \mathrm{~A}$ E Singh, ${ }^{4} \mathrm{~S}$ Drews, ${ }^{3} \mathbf{R}$ R Read. ${ }^{1}$ Alberta Health Services- STI Services, Edmonton, AB, Canada; ${ }^{2}$ Alberta Health Services- Edmonton STI Clinic, Edmonton, $A B$, Canada; ${ }^{3}$ Alberta Health Services- Calgary STI Clinic, Calgary, AB, Canada; ${ }^{4}$ Provincial Laboratory for Public Health-Calgary, Calgary, AB, Canada

Background In July 2012, two Alberta Sexually Transmitted Infection (STI) Clinics changed their testing platform for rectal chlamydia (CT) from cell culture to nucleic acid amplification testing (NAAT). A significant increase in the proportion of rectal-only CT cases occurred after the introduction of NAAT $(1.9 \% ; \mathrm{n}=25$ preNAAT vs. $20.1 \%$; $=245$ post-NAAT, $P<0.001)$. We sought to examine the characteristics of rectal-only CT cases.

Methods All CT cases seen at two Alberta STI clinics between July 20 and December 31, 2012 were extracted from the provincial STI database. Variables included were demographics, clinical history, 
reasons for visit and site of infection. Cases positive from the rectum alone were compared with cases positive from urethra, cervix, vault and urine alone or in multiple sites including the rectum. CT testing was conducted with Genprobe Aptima by the Provincial Laboratory for Public Health. Univariate analysis was completed using Chi-square or Fisher's exact test and Mann-Whitney for continuous variables. Bivariate logistic regression, adjusted for gender, was completed using significant $(P>0.05)$ at the univariate level.

Results Twenty percent of all CT cases $(n=245)$ were diagnosed in the rectum only; females were more likely to be diagnosed with rectal-only CT $(24.6 \%)$ than males $(16.6 \% ; P=0.001)$. No cases of rectal-only CT were found among heterosexual men; therefore regression models were completed for women and men who have sex with men (MSM). Factors associated with rectal-only CT for women included older age (AOR $=1.05,95 \% \mathrm{CI}: 1.02$, 1.08), being tested at Clinic A (AOR = 3.0, 95\% CI: 1.8, 5.1), and being named as a contact to an STI (AOR $=0.3,95 \%$ CI: 0.1, 0.9). For MSM, being asymptomatic ( $\mathrm{AOR}=2.2,95 \% \mathrm{CI}$ : $1.2,4.1)$ remained significant.

Conclusions After the switch to NAAT testing for rectal CT, additional cases of CT were found among women and MSM. Differences between clinics are likely attributable to different screening practises for women.

\section{P3.017 CHLAMYDIA TRACHOMATIS REPEAT TESTING IN AUSTRALIA}

doi:10.1136/sextrans-2013-051184.0477

\begin{abstract}
${ }^{12} \mathbf{M}$ Lim, ${ }^{3} \mathrm{~W}$ Dimech, ${ }^{1,2} \mathrm{C}$ Van Gemert, ${ }^{4} \mathrm{D}$ Boyle, 'D McCarthy, ${ }^{1} \mathrm{E}$ Weaver, ${ }^{5} \mathrm{~B}$ Donovan, 1,2M Hellard. 'Burnet Institute, Melbourne, Australia; ${ }^{2}$ Monash University, Melbourne, Australia; ${ }^{3} N R L$, Melbourne, Australia; ${ }^{4}$ University of Melbourne, Melbourne, Australia; ${ }^{5}$ Kirby Institute, University of New South UK, Sydney, Australia
\end{abstract}

Background Current guidelines recommend that sexually active people aged under 25 are screened annually for Chlamydia. Those testing positive should be retested around 12 weeks later to detect re-infection. The Australian Collaboration for Chlamydia Enhanced Sentinel Surveillance (ACCESS) Laboratory Network has collected chlamydia testing data from 15 Australian public and private laboratories since 2008 . This study reviews the frequency of repeat testing for $\mathrm{C}$. trachomatis.

Methods Chlamydia test results and associated demographic data were extracted from participating laboratories' information systems, de-identified with a non-reversible unique code and sent to a central database using GRHANITE ${ }^{\circledR}$ software. Using the unique identifier, cases of multiple testing episodes from individuals were reviewed to determine the frequency of repeat testing.

Results 641,302 chlamydia test results were collected from 547,761 individuals during the calendar years 2008-2010; 49,655 (7.7\%) were positive. Overall, $9.6 \%$ individuals had multiple testing episodes, increasing to $23.4 \%$ among those with an initially positive result. The mean number of testing episodes per individual was 1.11 (range 1-29) and mean time between repeat tests was 201 days following negative samples but 95 days after a positive sample. Among individuals who had a repeat test, for those with a negative result $19.6 \%$ of repeat tests were performed within 42 days, $42.8 \%$ within 120 days and $86.0 \%$ within 13 months. This is compared with $41.9 \%$ ( $\leq 42$ days), $76.6 \%$ ( $\leq 120$ days) and $96.6 \%$ ( $\leq 13$ months) for repeat tests following an initially positive result.

Conclusion Individuals with positive test results were found to be re-tested more frequently and earlier than those with negative test results. However, less than one quarter of individuals who tested positive for chlamydia were re-tested and over $40 \%$ of these were re-tested too soon after initial diagnosis ( $<6$ weeks), risking a false positive test result.

\section{P3.018 DEVELOPMENT OF A C. TRACHOMATIS-SPECIFIC COMPETITIVE PGP3 ELISA}

doi:10.1136/sextrans-2013-051184.0478

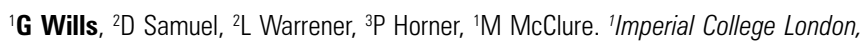
London, UK; ${ }^{2}$ Health Protection Agency, London, UK; ${ }^{3}$ University of Bristol, Bristol, UK

Background Chlamydia trachomatis (CT) DNA testing of genital samples principally from symptomatic persons provides information about active infection only, and is unlikely to represent true prevalence of current and past infection in the population. Serological tests applied to serum collections that are more representative of the general population can help understanding the pattern of the infection. We previously described an indirect immunoglobulin G (IgG) enzyme-linked immunosorbent assay (ELISA) based on the CT-specific antigen Pgp3. Sensitivity and specificity were determined using ROC curve analysis of data from 356 sera from CTinfected patients and 722 paediatric sera. The assay works particularly well in women, with a greater sensitivity $(74 \%)$ than commercial assays $(60 \%)$, and is suitable for use in seroprevalence studies. However, there is a need to confirm the specificity of samples reactive in the indirect Pgp3 ELISA and, to this end, we have developed a competitive Pgp3 ELISA.

Methods Purified IgG from human sera containing high titre antibody to CT was labelled with HRP and, by optimising conditions and using chequerboard titrations, an assay developed where test sera compete with labelled IgG for epitopes on the Pgp3 protein.

Results The competitive assay was optimised, then 89 sera from our CT-infected patient cohort (patients having had at least one positive CT NAAT result at least one month previously) and 91 paediatric sera were assayed by both the indirect and competitive Pgp3 ELISAs. Results by these two assays were concordant.

Conclusion A competitive ELISA based on the CT-specific Pgp3 protein has been developed, which confirms the specificity of the indirect Pgp3 ELISA.

\section{P3.019* IS CONCURRENCY, NUMBER OF PARTNERS OR DURATION OF PARTNERSHIP THE MOST IMPORTANT FACTOR ASSOCIATED WITH CHLAMYDIA IN YOUNG AUSTRALIAN ADULTS?}

doi:10.1136/sextrans-2013-051184.0479

${ }^{1}$ A Yeung, ${ }^{2} \mathrm{M}$ Temple-Smith, ${ }^{1} \mathrm{~A}$ Bingham, ${ }^{3,4} \mathrm{C}$ Fairley, ${ }^{5} \mathrm{M}$ Law, ${ }^{5} \mathrm{R}$ Guy, ${ }^{6} \mathrm{~N}$ Low, ${ }^{5} \mathrm{~B}$ Donovan, $5 \mathrm{~J}$ Kaldor, '1 Hocking. 'Centre for Women's Health, Gender \& Society, Melbourne School of Population and Global Health, Univ, Melbourne, Australia, ${ }^{2}$ Department of General Practice, University of Melbourn, Melbourne, Australia, ${ }^{3}$ Melbourne Sexual Health Centre, Melbourne, Australia, ${ }^{4}$ Melbourne School of Population and Global Health, University of Melbourne, Melbourne, Australia, ${ }^{5}$ Kirby

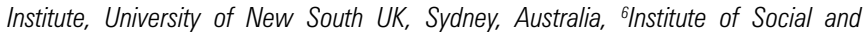
Preventive Medicine, University of Bern, Bern, Switzerland

Background There is considerable discussion about which sexual behaviour variables are most strongly associated with chlamydia. We investigated this in a study conducted within a chlamydia screening trial.

Methods A consecutive sample of patients aged 16-29 attending 134 GP clinics in 54 postcodes was recruited. Patients completed a questionnaire and chlamydia test. Using random effects logit regression models we estimated (1) the significance of a variable's association with chlamydia (likelihood ratio test for model fit), and; (2) the strength of association with chlamydia (odds ratio[OR]). Number of partners in the last 12 months and partnership duration (years) were fitted as continuous variables. Each model included age, gender and a sexual behaviour variable. A multivariate model including all sexual behaviour variables was also run. All analyses accounted for intra-cluster correlation within postcode. 